\title{
Myelination and isochronicity in neural networks
}

\author{
Fumitaka Kimura ${ }^{1 *}$ and Chiaki Itami ${ }^{2}$ \\ Department of Molecular Neuroscience, Osaka University Graduate School of Medicine, Japan \\ 2 Department of Physiology, Faculty of Medicine, Saitama Medical University, Moroyama, Saitama, Japan
}

\section{Edited by:}

Kathleen S. Rockland, RIKEN Brain

Science Institute, Japan

\section{Reviewed by:}

Giorgio Innocenti, Karolinska Institutet Sweden

Francisco Aboitiz, Pontificia Universidad

Catolica de Chile, Chile

*Correspondence:

Fumitaka Kimura, Department of Molecular Neuroscience, Osaka

University Graduate School of

Medicine, 2-2 Yamadaoka, Suita,

565-0871, Japan.

e-mail: fkimura@nphys.med.

osaka-u.ac.jp
Our brain contains a multiplicity of neuronal networks. In many of these, information sent from presynaptic neurons travels through a variety of pathways of different distances, yet arrives at the postsynaptic cells at the same time. Such isochronicity is achieved either by changes in the conduction velocity of axons or by lengthening the axonal path to compensate for fast conduction. To regulate the conduction velocity, a change in the extent of myelination has recently been proposed in thalamocortical and other pathways. This is in addition to a change in the axonal diameter, a previously identified, more accepted mechanism. Thus, myelination is not a simple means of insulation or acceleration of impulse conduction, but it is rather an exquisite way of actively regulating the timing of communication among various neuronal connections with different length.

\section{Keywords: isochronicity, thalamocortical pathway, conduction velocity, axon diameter}

\section{INTRODUCTION}

The timing when a neuron receives its incoming input has a great influence on how the input is processed and how it affects the postsynaptic neurons. On the other hand, an increase in the body size and the amount of information processed, as a result of evolution, inevitably required the expansion of the brain (Finarelli and Flynn, 2009; Jerison, 1955, 1961, 1973; Laughlin and Sejnowski, 2003). This resulted in situations where the excitation of a presynaptic neuron needs to arrive within a fixed window of time at target neurons located at multiple remote sites at variable distances. Such apparently paradoxical transmissions, in fact, take place in various regions of the brain. Some representative examples of such isochronicity can be observed in olivocerebellar connections (Lang and Rosenbluth, 2003; Sugihara et al., 1993), transcallosal connections in the visual cortex (Innocenti, 1995, 2009; Innocenti et al., 1994), amygdalocortical pathways (Pelletier and Pare, 2002), and still others that will be discussed later on. Intriguingly enough, these connections do not necessarily adopt the same strategy to accomplish isochronicity. Recently, we found that the thalamocortical pathway also exhibits the isochronic property, but with a novel mechanism, which involves differential myelination along the axon (Salami et al., 2003). Apparently this is an exquisite way of producing isochronicity and may apply to other systems. In this brief review, we will first survey how this is achieved in the thalamocortical pathway, and then discuss other isochronic pathways for comparison.

\section{ISOCHRONICITY IN THE THALAMOCORTICAL PATHWAY}

Neurons in the thalamus send axons to a wide area of the somatosensory cortex through different trajectories of various travelling lengths. Nevertheless, we reported that action potentials in the thalamic cells arrive almost simultaneously at each target cortical cell in layer IV. By using thalamocortical slices in which connection from thalamus to cortex were kept intact, EPSPs were recorded from layer IV cells in response to the stimulation of multiple sites such as thalamus, white matter (WM), and intracortical sites between WM and layer IV. This allowed us to measure the conduction velocity of each part of the axon while action potentials were travelling down the thalamocortical fibers. We found that the isochronicity in this system is achieved by changing the conduction velocity $(\mathrm{CV})$ within the individual axons. The $\mathrm{CV}$ decreases significantly upon entering the gray matter by up to 10 -fold (Salami et al., 2003). Originating from the thalamus, the axons of relay cells run in a straight path through the striatum up to the WM, at which point each axon diverges widely. Some ascend directly into the cortex up to layer IV, while others run in the subcortical WM for variable distances before ascending into the cortex. Although the total distances travelled vary across pathways, the distance of the intracortical regions is almost the same. That is, all the projections target the same layer IV in a cortex that has uniform thickness. The total conduction time primarily depends on the time spent within the cortex (gray matter). Thus, the strategy of making the $\mathrm{CV}$ of longer and variable parts by far faster than that of shorter and constant parts is a way of eliminating the variability in traversed distances (Figure 1).

Myelination is involved in this process in two-fold. First, since $\mathrm{CV}$ is proportional to the square root of the axon diameter in unmyelinated axons, to have 10 times larger $\mathrm{CV}$, axon diameter needs to be 100 times larger, which is most unlikely. This led us to predict that thalamocortical fibers would be myelinated. Thus, secondly, we suspected that the extent of myelin might cause the observed difference of CV. Histological staining of the myelin revealed that the difference of myelination between the intracortical and extracortical (WM) regions played an important role in the generation of CV difference. Developmentally, CV difference could be seen after the end of the 2nd postnatal week. At this time there is practically no myelination observed in the grey matter, while thalamus to white matter portions are already heavily myelinated (Salami et al., 2003). It appeared that such differential myelination continued in 


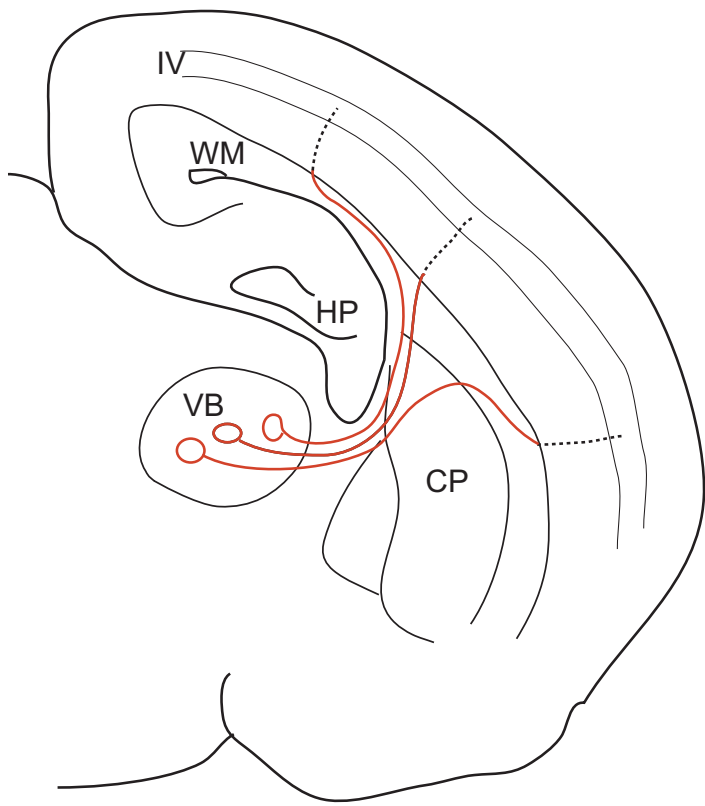

FIGURE 1 | A schematic illustration of the thalamocortical pathway, showing regional differences in $\mathbf{C V}$. By having a 10-fold faster $\mathrm{CV}$ from the VB to the WM (red lines), most of the conduction time is spent on the intracortical regions (dotted lines), whose length is generally constant due to the homogeneous structure of the cortex. Thus, isochronicity of conduction time from VB to layer IV cells is achieved. VB: ventrobasal nucleus of thalamus, WM: white matter, HP: hippocampus, CP: caudate putamen, IV: layer 4 [Cited from Salami et al., PNAS 100, 6174-6179 (2003)].

the adulthood. Since this was only a light microscopic observation, EM-level confirmation remains to be done, however.

This intra-axonal, segmental difference is well-suited to influence the difference in CV. Thus, we hypothesized that difference of myelination plays a major role in creating isochronicity. In other words, myelination is not merely insulation among neighboring cells, but is a method of regulating the timing of postsynaptic activations. A change in the $\mathrm{CV}$ along a given axon has been reported in other regions of the brain. The axons of retinal ganglion cells increase their CV from the optic nerve to the optic tract at the point of the optic chiasm, by increasing both their diameter and myelination (Baker and Stryker, 1990). Similarly in the peripheral nervous system, A $\delta$ fibers supplying mechanoreceptors exhibit serious reduction in the CV upon entering the spinal cord (Traub and Mendell, 1988). In addition to sensory neurons, motoneurons of the ventral horn increase their sheath thickness and axon diameter upon exiting the spinal cord (Fraher, 1976, 1978). Our results indicate that these changes in $\mathrm{CV}$ and the associated anatomical changes may well play a role in the regulation of activation timing.

\section{ISOCHRONICITY IN OTHER REGIONS IN THE BRAIN}

Isochronicity has been reported in other regions of the brain. In rats, axons of the inferior olive innervate widely distributed regions of the cerebellar cortex. The conduction time to the different parts of the cerebellum is relatively invariant despite differences in path length. The isochronicity in the olivocerebellar projection is the result of a differential CV. An earlier study has shown that longer fibers conduct faster than shorter ones due to differential axon diameters rather than differential myelination (Sugihara et al., 1993). A recent study challenged this view, claiming that myelination plays a primary role in generating a uniform olivocerebellar conduction time (Lang and Rosenbluth, 2003). Interestingly, it has been questioned whether this invariance holds for larger animals, such as cats (Aggelopoulos et al., 1995), although even in this study the latency of olivocerebellar projections varied only by several milliseconds.

Isochronic property of transcallosal connections is one of the most intensively studied within the framework of the computational properties of axons by Innocenti and his group (Innocenti, $1995,2009)$. Based on the simulation of action potential propagation determined by axonal diameter, these authors provided a detailed description of strategies for callosal connections to achieve synchronous activation of their targets (Innocenti et al., 1994).

In the cortex, layer $\mathrm{V}$ pyramidal neurons project to various subcortical regions as well as to the contralateral side. One recent study showed that layer $\mathrm{V}$ pyramidal neurons in the ventral temporal lobe innervate diverse regions such as the caudate putamen, parietal cortex, amygdala, and thalamic nuclei on the ipsilateral side with isochronic spike delivery based on the differential CVs in each fiber branch (Chomiak et al., 2008). By combining the actual measurement of the axonal inner and outer diameters, with theoretical predictions based on partial myelination, the observation of Chomiak et al. on isochronicity appears to be best supported by partial or differential myelination. It may be worth mentioning that the same neurons send axons to the contralateral side through the corpus callosum, but the CVs were significantly slow and not isochronic with ipsilateral connections.

The lateral amygdala is believed to play an important role in establishing fear memory in cooperation with the perirhinal cortex through Hebbian synaptic interactions, where the timing of synaptic input is important. The perirhinal cortex is an elongated structure as compared to the rather small nucleus of the lateral amygdala. Electrophysiological experiments have revealed that the lateral amygdala is isochronically connected with a large portion of perirhinal cortex (Pelletier and Pare, 2002), but the mechanism of this isochronic connection is yet to be elucidated.

\section{ISOCHRONICITY IN THE DEVELOPING BRAIN}

Although brain and body sizes increase during development, the conduction times of certain connections remain constant. One study in humans has revealed that the somatosensory and motor conduction times remain constant even after 2 years of age, despite substantial increases in the axon length that occur with body growth (Eyre et al., 1991). Experiments in animals investigated the rubrospinal tract in cats (Song et al., 1995), and revealed that the conduction time from the red nucleus to L1 reached the minimum adult level at P30, when the spinal cord is only half the adult length. CV increased linearly from E59 to P30 (from 1-34 m/s). Since myelination of the rubrospinal tract was believed to have begun before E59, the adult level conduction time was achieved mainly by myelination until P30, then thereafter, axon diameter was increased so as to keep the constant conduction time as the axon length increased. Thus, these authors proposed that compensatory interaction of 
myelination and axon diameter led to the isochronic conduction during development.

In other systems, however, myelination is unlikely to be involved in isochronicity during development. The locus coeruleus (LC) in the dorsal pons sends axons widely throughout the brain including the frontal cortex, but latencies remain constant throughout life (Nakamura et al., 1987), even when the size of the brain increases to more than 2 times its original size. Since axons of the LC consist of C fibers lacking myelin, mechanism(s) other than myelination should account for the associated increase in the CV.

\section{ISOCHRONICITY AND ACTIVITY-DEPENDENT MYELINATION}

One implication from these studies is that each pathway has its own conduction time, which might be determined by its function. Myelination, axon diameter, and other factors such as the structure, or the number of spacing of Ranvier nodes, and ion channel composition and/or its density, all of these would affect the $\mathrm{CV}$, and conduction time may be employed to achieve its characteristic value. On the other hand, since the environment around a given neuron, or tissue, or whole individual continuously changes, a regulatory mechanism that changes in an input- or activity-dependent manner is desirable to adapt to the changing environment.

Myelination indeed changes in an experience- or inputdependent manner. In dark-reared mice, the number of myelinated axons in the optic nerve decreased (Gyllensten and Malmfors, 1963). Similarly, premature eye opening in rabbit reduced the expression of myelin (Tauber et al., 1980). A diffusion tensor imaging study revealed that extensive piano practicing in childhood results in a thicker white matter, which is believed to be due to a change in myelination (Bengtsson et al., 2005). Several lines of evidence have identified how action potentials regulate myelination. A specific pattern of neural activity was shown to lower the expression of L1-CAM (Itoh et al., 1995) that is necessary for myelin induction by oligodendrocytes (Barbin et al., 2004), as well as by Schwann cells (Stevens et al., 1998). In addition, ATP released from axon terminals as a result of neural activities facilitates the differentiation of oligodendrocytes through adenosine and $\mathrm{P} 1$ receptors. For astrocytes, ATP causes the release of leukemia inhibitory factor (LIF), which then stimulates myelination by oligodendrocytes (Ishibashi et al., 2006). A classical view on myelin thickness is that the thickness of myelin sheath is closely related with the axon diameter. Theoretically, optimal or fastest $\mathrm{CV}$ is provided when $g$-ratio is 0.65 , where $g$-ratio is defined as the axon diameter divided by total fiber diameter. Although it is generally true that axons conforming to this $g$-ratio is widely observed, it is also true that there are still others with large variation in $g$-ratio around the mean, and considerable differences are seen between fibers (Berthold et al., 1983). In fact, optic nerve axons have uniform diameters, but exhibits five different CVs. These are ascribed to as many differences in myelination (Freeman, 1978). Similarly, as mentioned before, multiple CVs along the same fiber were reported from other studies (Baker and Stryker, 1990; Traub and Mendell, 1988). If as we discussed earlier, each pathway has its own conduction time, and if myelination changes in an inputdependent manner to adapt to environment, it seems reasonable to propose that the difference of the extent of myelination may be the result of adaptation whereby myelination influences its own conduction time.

\section{FUNCTIONAL SIGNIFICANCE OF ISOCHRONICITY}

What is the functional significance of such isochronicity of action potential conduction? The issue of isochronicity has actually a long history and has been demonstrated also in invertebrates. Among the oldest of these examples are the classical studies on the squid giant axons. Conduction time of the giant axons between the stellate ganglia and the mantle musculature is such that activation of the whole mantle takes place synchronously, allowing effective escape to occur. This is achieved by faster CV for longer axons, by changing axon diameters (Young, 1939) exclusively. That is because invertebrates do not have myelination with which the CV could be enormously ( $\sim 100$ times) increased. In other words, vertebrates are endowed with an additional mechanism in regulating $\mathrm{CV}$. It appears quite reasonable to think that vertebrates would exploit these strategies independently. In electric organs of a certain kind of fish, synchronous activation of electrocytes to produce larger electrical potentials is attained by two mechanisms; first, same as squid axon, faster CV for longer pathways, and secondly, longer circuitous route for cells closer to the target (Bennett, 1970). The avian auditory system is another similar example where circuitous routes are used to compensate for shorter distance. The conduction time between the cochlear nucleus and its ipsi- and contralateral nucleus laminaris is matched by lengthening the path to the ipsilateral nucleus, to create a delay line for detecting the differential timing of sound inputs to both ears for sound localization. In these cases, the advantage of the isochronous conduction is obvious.

By analogy, in those connections we have discussed above, such as olivocerebellar, amygdalo-perirhinal, and corticofugal from ventral temporal cortex, isochronous activations of target cells should have clear temporal advantages. However, at this point, how isochronicity is advantageous does not seem obvious in these systems. One interesting view is that the olivocerebellar system serves as an intrinsic timing device that is essential for motor co-ordination (Llinas, 1988). This view is based on the observation that neurons in the inferior olive are connected by gap junctions and thus exhibit oscillatory activity. This is supposed to propagate to a wide territory of the cerebellar cortex, a process for which isochronicity is essential.

A similar situation might apply to the thalamocortical pathway. Thalamic cells also show oscillatory activities intrinsically (Contreras et al., 1996) that could cause synchronization in the functionally related target cortical cells. Isochronous or synchronous activation of group of cells distributed in distant cortical locations with zero phase-lag have been shown in visual cortex (Gray et al., 1989), and callosally connected areas (Engel et al., 1991). These findings attracted attention as relevant to the problem of connecting features responsible for object recognition in the spatially fractured nature of sensory representation over the cortical mantle (Llinas et al., 2002; Singer, 1999) and providing the perception of unity (von der Malsburg and Buhmann, 1992). For such binding to work correctly, isochronous activation of related cells in the global cortical area would be a necessary condition. 
Isochronous activation of thalamocortical as well as transcallosal connections provides a physiological and anatomical explanation for these observations. Synchronous activities may also be appropriate for producing synfire chains (Abeles, 1991). Another possible explanation for the resultant isochronicity might be, as we discussed before, that each connection has its own characteristic conduction time for information to be correctly processed in the network. Under such conditions, if postsynaptic target neurons are widely distributed, isochronous conduction will result. This is in some sense consistent with isochronicity in the developing brain as we described earlier. During development, once a pathway with a characteristic conduction time becomes functional, the specific conduction time presumably needs to be kept constant in spite of the increasing distances subsequent to growth of the body. The corpus callosum displays a significant variety in terms of myelination (Aboitiz et al., 1992). In rats, the fraction of myelinated fibers is zeroe at birth, then it gradually increases to $53 \%$ at 300 days post-conception (Seggie and Berry, 1972). Similarly in humans, none of the callosal fibers are myelinated at birth, and in adults $30 \%$ of the fibers remain unmyelinated. In addition, analyses of fiber composition revealed a wide variety of fiber diameters and extent of myelination depending on the target area. Callosal regions connecting prefrontal and temporoparietal association areas consist of small caliber with low myelinated fibers, whereas regions connecting primary and secondary sensorimotor areas include highly myelinated, large-caliber fibers (Aboitiz et al., 2003). Consequently, the conduction time between two hemispheres varies from $30 \mathrm{~ms}$

\section{REFERENCES}

Abeles, M. (1991). Corticonics: Neural Circuits of the Cerebral Cortex. Cambridge, Cambridge University Press.

Aboitiz, F., Lopez, J., and Montiel, J. (2003). Long distance communication in the human brain: timing constraints for inter-hemispheric synchrony and the origin of brain lateralization. Biol. Res. 36, 89-99.

Aboitiz, F., Scheibel,A. B., Fisher, R. S., and Zaidel, E. (1992). Fiber composition of the human corpus callosum. Brain Res. 598, 143-153.

Aggelopoulos, N. C., Duke, C., and Edgley, S. A. (1995). Non-uniform conduction time in the olivocerebellar pathway in the anaesthetized cat. J. Physiol. 486, 763-768.

Baker, G. E., and Stryker, M. P. (1990). Retinofugal fibres change conduction velocity and diameter between the optic nerve and tract in ferrets. Nature 344, 342-345.

Barbin, G., Aigrot, M. S., Charles, P., Foucher,A.,Grumet,M.,Schachner, M., Zalc, B., and Lubetzki, C. (2004). Axonal cell-adhesion molecule L1 in CNS myelination. Neuron Glia Biol. 1, 65-72.

Bengtsson, S. L., Nagy, Z., Skare, S., Forsman, L., Forssberg, H., and

Chomiak, T., Peters, S., and Hu, B. (2008).

via myelinated axons to as long as $300 \mathrm{~ms}$ via unmyelinated ones (Fields, 2008). Since callosal fibers connect a variety of cortical regions with various functions, conduction times for each functions are likely to be diverse. The extent of myelination, as well as axon diameter might help regulate the conduction time to its optimal value for communicating between hemispheres. This leads to another interesting question; namely, how the conduction time of each pathway is determined in the network. Overall, our understanding regarding the time devoted to each step in neural processing is still, unfortunately, severely limited.

\section{CONCLUSION}

Isochronic propagation of information in spite of variable neuronal distances eliminates the limitation of connectional distances, and is permissive for the expansion of the brain. This phenomenon is seen widely in various brain regions, and at several phylogenetic levels, as well as during development. Isochronicity is achieved mainly by differential CVs, either within individual axons or among axons. It is suggested that the vertebrates obtained another option in generating differential CVs, that is, changing myelination, in addition to regulating axon diameters.

\section{ACKNOWLEDGMENTS}

This work was partly supported by Grants in Aid for the Ministry of Education, Culture, Sports, Science and Technology (F.K, C.I). Authors are grateful to Drs. H. Sawai and T. Miyoshi for helpful discussions.

somatosensory pathways. J. Physiol. ticing has regionally specific effects on white matter development. Nat. Neurosci. 8, 1148-1150.

Bennett, M. V. (1970). Comparative physiology: electric organs. Annu. Rev. Physiol. 32, 471-528.

Berthold, C. H., Nilsson, I., and Rydmark, M. (1983). Axon diameter and myelin sheath thickness in nerve fibres of the ventral spinal root of the seventh lumbar nerve of the adult and developing cat. J. Anat. 136, 483-508. Functional architecture and spike timing properties of corticofugal projections from rat ventral temporal cortex. J. Neurophysiol. 100, 327-335.

Contreras,D., Destexhe,A.,Sejnowski, T. J., and Steriade, M. (1996). Control of spatiotemporal coherence of a thalamic oscillation by corticothalamic feedback. Science 274, 771-774.

Engel, A. K., Konig, P., Kreiter, A. K., and Singer, W. (1991). Interhemispheric synchronization of oscillatory neuronal responses in cat visual cortex. Science 252, 1177-1179.

Eyre, J. A., Miller, S., and Ramesh, V. (1991). Constancy of central conduction delays during development in man: investigation of motor and

Finarelli, J. A., and Flynn, J. J. (2009). 434, 441-452.

quantitative investigation in mice. Embryol. Exp. Morphol. 11, 255-266.

Fields, R. D. (2008). White matter in learning, cognition and psychiatric disorders. Trends Neurosci. 31, 361-370. Brain-size evolution and sociality in Carnivora. Proc. Natl. Acad. Sci. U.S.A. 106, 9345-9349.

Fraher, J.P. (1976). The growth and myelination of central and peripheral segments of ventral motoneurone axons. A quantitative ultrastructural study. Brain Res. 105, 193-211.

Fraher, J. P. (1978). Quantitative studies on the maturation of central and peripheral parts of individual ventral motoneuron axons. I. Myelin sheath and axon calibre. J. Anat. 126, 509-533.

Freeman, B. (1978). Myelin sheath thickness and conduction latency groups in the cat optic nerve. J. Comp. Neurol. 181, 183-196.

Gray, C. M., Konig, P., Engel, A. K. and Singer, W. (1989). Oscillatory responses in cat visual cortex exhibit inter-columnar synchronization which reflects global stimulus properties. Nature 338, 334-337.

Gyllensten, L., and Malmfors, T. (1963). Myelinization of the optic nerve and its dependence on visual function - a
Innocenti, G. M. (1995). Exuberant development of connections, and its possible permissive role in cortical evolution. Trends Neurosci. 18, 397-402.

Innocenti, G. M. (2009). Dynamic interactions between the cerebral hemispheres. Exp. Brain Res. 192, 417-423.

Innocenti, G. M., Lehmann, P., and Houzel, J. C. (1994). Computational structure of visual callosal axons. Eur. J. Neurosci. 6, 918-935.

Ishibashi, T., Dakin, K. A., Stevens, B., Lee, P. R., Kozlov, S. V., Stewart, C. L., and Fields, R. D. (2006). Astrocytes promote myelination in response to electrical impulses. Neuron 49, 823-832.

Itoh, K., Stevens, B., Schachner, M., and Fields, R. D. (1995). Regulated expression of the neural cell adhesion molecule L1 by specific patterns of neural impulses. Science 270, 1369-1372.

Jerison, H. J. (1955). Brain to body ratios and the evolution of intelligence. Science 121, 447-449. of evolution of the brain in mammals. Science 133, 1012-1014.
Jerison, H. J. (1961). Quantitative analysis 
Jerison, H. J. (1973). Evolution of the Brain and Intelligence. New York, Academic Press.

Lang, E. J., and Rosenbluth, J. (2003). Role of myelination in the development of a uniform olivocerebellar conduction time. J. Neurophysiol. 89, 2259-2270.

Laughlin, S. B., and Sejnowski, T.J. (2003). Communication in neuronal networks. Science 301, 1870-1874.

Llinas, R. R. (1988). The intrinsic electrophysiological properties of mammalian neurons: insights into central nervous system function. Science 242 , 1654-1664.

Llinas, R. R., Leznik, E., and Urbano, F. J. (2002). Temporal binding via cortical coincidence detection of specific and nonspecific thalamocortical inputs: a voltage-dependent dye-imaging study in mouse brain slices. Proc. Natl. Acad. Sci. U.S.A. 99, 449-454.

Nakamura,S.,Kimura,F., and Sakaguchi, T. (1987). Postnatal development of elec- trical activity in the locus ceruleus. $J$. Neurophysiol. 58, 510-524.

Pelletier,J.G., and Pare, D. (2002).Uniform range of conduction times from the lateral amygdala to distributed perirhinal sites. J. Neurophysiol. 87, 1213-1221.

Salami, M., Itami, C., Tsumoto, T., and Kimura, F. (2003). Change of conduction velocity by regional myelination yields constant latency irrespective of distance between thalamus and cortex. Proc. Natl. Acad. Sci. U.S.A. 100, 6174-6179.

Seggie, J., and Berry, M. (1972). Ontogeny of interhemispheric evoked potentials in the rat: significance of myelination of the corpus callosum. Exp. Neurol. $35,215-232$.

Singer, W. (1999). Neuronal synchrony: a versatile code for the definition of relations? Neuron 24, 49-65, 111-125.

Song, W. J., Okawa, K., Kanda, M., and Murakami, F. (1995). Perinatal development of action potential propa- gation in cat rubrospinal axons. $J$ Physiol. 488, 419-426.

Stevens, B., Tanner, S., and Fields, R. D. (1998). Control of myelination by specific patterns of neural impulses. J. Neurosci. 18, 9303-9311.

Sugihara, I.,Lang,E.J., and Llinas, R. (1993). Uniform olivocerebellar conduction time underlies Purkinje cell complex spike synchronicity in the rat cerebellum. J. Physiol. 470, 243-271.

Tauber,H., Waehneldt, T.V., and Neuhoff, V. (1980). Myelination in rabbit optic nerves is accelerated by artificial eye opening. Neurosci. Lett. 16, 235-238.

Traub, R. J., and Mendell, L. M. (1988). The spinal projection of individual identified A-delta- and Cfibers. J. Neurophysiol. 59, 41-55.

von der Malsburg, C., and Buhmann, J. (1992). Sensory segmentation with coupled neural oscillators. Biol. Cybern. 67, 233-242.

Young, J. Z. (1939). Fused neurons and synaptic contacts in the giant nerve fibres of cephalopods. Philos. Trans. R. Soc. Lond. B Biol. Sci. 299 , 465-503.

Conflict of InterestStatement:Theauthors declare that the research was conducted in the absence of any commercial or financial relationships that could be construed as a potential conflict of interest.

Received: 01 May 2009; paper pending published: 15 May 2009; accepted: 23 June 2009; published online: 06 July 2009.

Citation: Kimura F and Itami $C$ (2009)

Myelination and isochronicity in neural networks. Front. Neuroanat. (2009) 3:12. doi:10.3389/neuro.05.012.2009

Copyright $\odot 2009$ Kimura and Itami. This is an open-access article subject to an exclusive license agreement between the authors and the Frontiers Research Foundation, which permits unrestricted use, distribution, and reproduction in any medium, provided the original authors and source are credited. 